\title{
Contaminación biológica y otros factores de riesgo relacionados con el desempeño en los laboratorios de docencia de la Universidad Colegio Mayor de Cundinamarca en Santa Fe de Bogotá
}

\begin{abstract}
Alicia Alvarez, Silvia Campuzano
Programa de Bacteriologia, Universidad Colegio Mayor de Cundinamarca, Santa Fe de Bogotá, D.C., Colombia.

Preocupados por el avance científico y tecnológico que involucra directamente el desempeño de los laboratorios y que continúa generando riesgos potenciales para la salud de los trabajadores y el medio ambiente, surgió la necesidad de realizar un diagnóstico para el programa de bacteriología que permitiera la identificación de los riesgos y la elaboración de un panorama de riesgos propios $y$, a su vez, proponer acciones preventivas frente a riesgos específicos. Como una institución de formación de recurso humano en salud, específicamente en bacteriología, y para estar acorde con las políticas nacionales de promoción y prevención, es fundamental formar una nueva cultura de prevención de los riesgos. Esta investigación fortalece acciones propuestas en el Programa de Salud Ocupacional institucional, como es el establecimiento del panorama de riesgos mediante la identificación de factores de riesgos ocupacionales relacionados con el desempeño en el laboratorio. El estudio se desarrolló a partir del reconocimiento y análisis de las áreas que se iban a investigar, seguido por la valoración de la contaminación de los laboratorios, la clasificación de los factores de riesgos ocupacionales y, por ende, la determinación del panorama de riesgos propio de la Facultad de Ciencias de la Salud. Esta problemática comprometía a un gran núcleo social de la universidad, y es así como para su desarrollo se aplicaron métodos participativos: la observación participante, la encuesta de conocimientos, actitudes y prácticas y la entrevista semiestructurada. Todos son métodos encaminados a sensibilizar y a concientizar a la comunidad. Se encontró que el riesgo biológico era el de mayor incidencia, sustentado por el análisis microbiológico que informó la presencia de microorganismos patógenos como Streptococcus pyogenes, Pseudomonas sp. y levaduras, entre otros. En su orden, siguieron el riesgo psicosocial, químico, físico, de seguridad y ergonómico. Se obtuvo como resultado el panorama de riesgos propio, que permite establecer la prevención frente al riesgo y que será interiorizada por la comunidad mediante el desarrollo de un programa educativo y verificado por un sistema de vigilancia epidemiológica.
\end{abstract}

Palabras clave: riesgos ocupacionales, prevención, factores de riesgo, panorama de riesgos.

Biological contamination and other risk factors concerning teaching labotatory performance at Mayor de Cundinamarca University in Santa Fe de Bogotá

Scientific and technological advances directly involving the performance of laboratory tasks keep being a source of potential risks for laboratory workers' health and for the environment. The need to carry out an assessment of the bacteriology program emerged from this concern. The evaluation allowed us not only to identify risks and, consequently, to elaborate a panorama of those specific to the program, but also to suggest prevention measures for specific risks. As an institution devoted to training health workers -particularly in the field of bacteriology-, and committed with national promotion and prevention policies, it is paramount for us to educate towards a new culture of risk prevention. This research supports actions proposed for the University's occupational health program, such as elaborating the panorama of risks by identifying the occupational health risk factors related to laboratory work. 
The study started with the identification and analysis of the research areas. This task was followed by an assessment of contamination levels in the laboratories, as well as by a classification of occupational risk factors, so that a panorama of specific risks at the Health Sciences School could be established. This problem involved a wide social sector of the university. Therefore, participatory methods were applied during the development of the research. The methods used were: participating observation; knowledge, attitude and practice survey, and semiestructured interview. All of these methods were addressed to promote the community's sensitivity and awareness. The highest incidence was found in biological risk. This finding was supported by the microbiological analysis, which reported the presence of microorganisms such as Streptococcus pyogenes, Pseudomona and yeasts. Psychosocial, chemical, physical, safety, and ergonomic risks followed in incidence. The result of the study was a panorama of specific risks of the bacteriology program, which allows the implementation of prevention measures. These will be internalized by the community by means of an educational program, and verified by an epidemiological surveillance system.

Key words: occupational risks, prevention, risk factors, panorama of risks.

Dentro del esquema de análisis en salud ocupacional de los diferentes riesgos que afectan al personal que labora en las instituciones, cabe resaltar la importancia que cobran áreas selectivas que involucran una situación crítica, como son los laboratorios de diagnóstico y de producción (1).

A diferencia de otras ocupaciones, los riesgos 0 accidentes de trabajo en los laboratorios de salud pueden evitarse con solo observar cuidadosamente las medidas o normas de seguridad en las diferentes áreas de acción, las cuales, a pesar de que son herramientas que permiten la realización del trabajo con mayor confianza y seguridad, no son observadas por el personal de los laboratorios, debido a una serie de factores como la desinformación, la negligencia, la ignorancia y la inexperiencia, entre otros (1).

Es así como se detecta la necesidad de disminuir y prevenir estos riesgos que se resumen en biológicos, físicos, químicos, ergonómicos y psicosociales. Por tanto, la principal razón de este estudio fue la de hacer el diagnóstico de los factores de riesgo y proponer acciones de prevención aplicables al trabajo diario en los laboratorios de práctica de un programa de bacteriología, para así poder establecer el panorama de riesgos propios y el programa educativo que alcance el logro de una cultura de

$\overline{\text { Correspondencia: }}$

Alicia Alvarez, Carrera 30 No.22-A-08, Torre 2, Apartamento 905, Santa Fe de Bogotá, D.C., Colombia

Recibido: 21/12/99; aceptado: 07/04/00 prevención frente a ellos. Para esto, es fundamental la educación centrada en tres componentes: información, formación y motivación, que deben ser dirigidos hacia las diferentes poblaciones de manera continua y permanente (2).

La universidad es un semillero del saber y, por ello, es el sitio ideal para lograr la capacitación y formación adecuada de los profesionales de la salud, en este caso de los bacteriólogos, en cuanto al manejo de material biomédico de mucha peligrosidad; al igual que inculcarles la cultura de prevención que debe guiarlos en el desempeño de su vida profesional y personal y prepararlos para que se comprometan con el autocuidado y puedan liderar campañas de promoción y prevención de la salud en la comunidad con que interactúan.

Para comprometer a la comunidad involucrada en la solución de su problemática, se estructuró un modelo de evaluación basado en métodos cualitativos como la observación participante y la entrevista semiestructurada, cuyos datos suministran descripciones detalladas de las situaciones, interacciones y comportamientos observados (2).

Para reforzar estos métodos, se aplicó una dinámica cuantitativa, que proporciona datos validados estadísticamente como la encuesta de conocimientos, actitudes y prácticas (CAP) en la cual, a partir del conocimiento, se obtiene la comprensión y análisis de una pregunta concreta que exige una respuesta igual; explorando las 
actitudes, se sabe qué sustentan los valores, propósitos, preferencias, prejuicios y convicciones prácticas, y el comportamiento de la población en estudio, representado por conductas, procedimientos y hábitos, permite evaluar todo lo que rodea al sujeto y de lo que participa (3).

Todos estos datos se analizaron a la luz de la triangulación de la información, con el fin de proteger las tendencias de los investigadores y de confrontar y someter a control recíproco las perspectivas de diferentes personas, para así obtener una comprensión más profunda y clara del escenario y de las personas objeto del estudio (4).

Se concluyó que el riesgo biológico ocupa lugar preponderante entre los factores que deben prevenirse con mayor prioridad. En segunda instancia, aparece el riesgo psicosocial que se encuentra relacionado directamente con la responsabilidad intrínseca e individual dentro del trabajo en el laboratorio.

Con el fin de mejorar y corregir la situación detectada, se propuso un modelo educativo flexible e innovador como instrumento de formación para la prevención, que involucre la construcción de conocimiento hacia la transformación del quehacer diario.

Es fundamental nombrar un comité de control que pueda desarrollar acciones de intervención por medio de un programa de vigilancia epidemiológica que evalúe y retroalimente permanentemente el proceso.

\section{Materiales y métodos}

\section{Tipo de estudio y población}

Se realizó un estudio de tipo descriptivo; la población estudiada fue de 162 personas que incluían directivas, docentes, estudiantes y personal de servicios generales del programa de bacteriología de la Universidad Colegio Mayor de Cundinamarca. El estudio se desarrolló entre febrero de 1997 y julio de 1999 y se llevó a cabo en tres etapas: en la primera se practicó el levantamiento del diagnóstico, en la segunda se clasificaron los riesgos y se diseñó su panorama y en la tercera se propusieron las medidas de prevención.

\section{Levantamiento del diagnóstico}

Se realizó con el reconocimiento y análisis de las áreas que se iban a investigar, la valoración de la contaminación y la identificación y clasificación de los riesgos. Se analizaron 15 laboratorios que comprendian un laboratorio central, 11 laboratorios de práctica, un bioterio, un laboratorio de micología y un laboratorio de investigación, mediante la observación analítica de espacios (5). La observación se realizó con base en adecuación de espacios, organización y distribución de elementos, limpieza de mesones, equipos y disponibilidad de elementos para desechos y manejo de ellos.

\section{Valoración de la contaminación}

En los 11 laboratorios de práctica, se realizaron cultivos para bacterias y hongos de paredes, mesones, equipos, vertederos y ambiente; en el laboratorio central, se estudiaron las áreas de cepario, cuarto frío, esterilización, materiales y ambiente.

En el laboratorio de micología y de investigaciones, no se realizó valoración de contaminación porque en el análisis de la observación no se encontraron deficiencias. En cuanto al bioterio, se estableció que debe adecuarse desde la estructura física, razón por la cual no se tuvo en cuenta para la valoración de la contaminación.

El algoritmo que se aplicó para el análisis de la contaminación de los laboratorios, se observa en la figura 1. La toma de muestras, los cultivos realizados y los métodos diferenciales se adaptaron de la norma de microbiología para cultivos de ambiente y superficies (6-8). Los resultados de los cultivos se expresaron en porcentaje de la muestra estudiada.

Los cultivos para bacterias y hongos se practicaron en dos fases: la primera fase, al inicio del semestre, momento en que se ha practicado limpieza y desinfección total de las áreas físicas, del ambiente y de los diferentes elementos de los laboratorios. El proceso de limpieza comprende desinfección con hipoclorito de sodio $(0,5$ a $1 \%)$ en el caso de mesones, paredes y vertedero; desinfección varias veces con alcohol al $70 \%$ en el caso de equipos como microscopios; lavado 


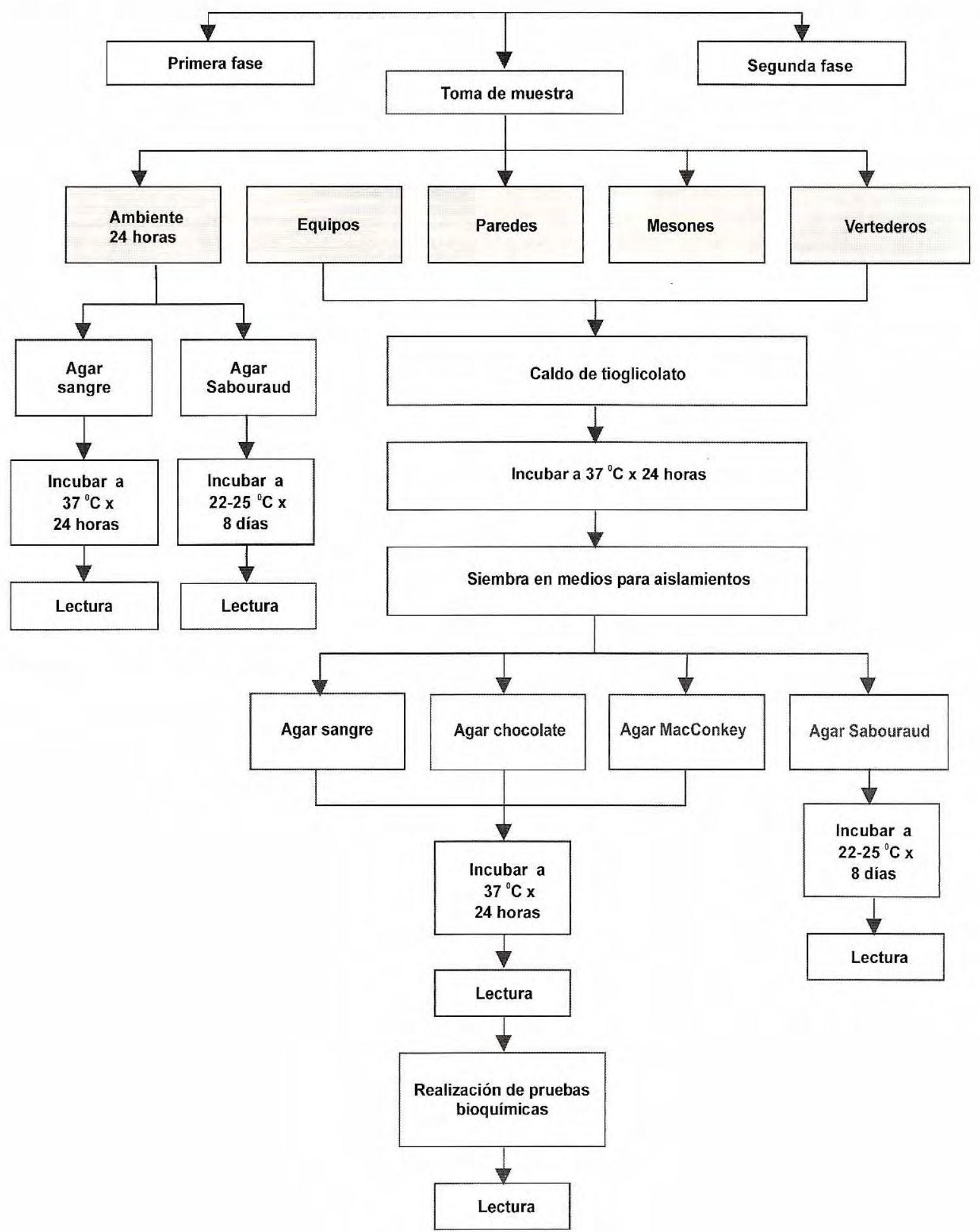

Figura 1. Algoritmo para el análisis de la contaminación de los laboratorios. 
con agua y jabón para el piso, mesones y vertederos; nueva limpieza con hipoclorito de sodio $(0,5$ a $1 \%)$ al piso, mesones y vertederos. La segunda fase se realizó finalizando el semestre, cuando las clases prácticas están en pleno desarrollo y se han manipulado todo tipo de microorganismos y muestras biológicas.

La identificación y clasificación de los riesgos biológicos, psicosociales, químicos, físicos, de seguridad y ergonómicos propios del desempeño en los laboratorios de práctica se realizó mediante el empleo de tres métodos diseñados para investigación cualitativa (8), como son la observación participante, las encuestas CAP y la entrevista semiestructurada $(9-12)$.

Para la observación participante, se diseñó la guía de observación, teniendo como referencia las normas de bioseguridad establecidas para cada riesgo; luego, se practicaron actividades de sensibilización a la comunidad involucrada para generar confianza y obtener un resultado cercano a la realidad cotidiana; se desarrolló la observación en el sitio de práctica diligenciando la guía.

Para la medición se utilizó la escala de 'siempre', 'casi siempre', 'casi nunca' y 'nunca'. La interpretación se realizó con porcentajes. En el caso del método cuantitativo, o sea la encuesta CAP, se plantearon los objetivos, la justificación y metas propias de la encuesta para poder diseñar el cuestionario que debe contener preguntas relacionadas con los conocimientos, actitudes y prácticas en el desarrollo del trabajo en los laboratorios de diagnóstico de la universidad. Luego, se valoraron los resultados mediante la aplicación de métodos estadísticos para obtener las frecuencias correspondientes a cada pregunta.

Para el otro método cualitativo, la entrevista semiestructurada, se revisaron y analizaron los resultados de los dos métodos anteriores, con el fin de encontrar vacíos no cubiertos anteriormente; se elaboró una guía flexible que reforzara los aspectos faltantes o no claros en los métodos aplicados. Igualmente, se desarrolló sensibilización para obtener resultados más confiables.

Para el desarrollo de la entrevista, se realizaron reuniones con grupos focales de más o menos 12 personas. La recolección de la información, además de recopilar los comentarios por escrito, se grabó en cassettes de audio para luego elaborar la bitácora correspondiente que permitiera la interpretación y el análisis de los resultados.

Los resultados de estos tres métodos se integraron mediante la triangulación de la información, en la cual figuran como elementos principales la realidad con las personas, sus experiencias y percepciones, que surgen en los registros de observación y entrevistas representados en el texto descriptivo y que demuestran la apariencia del objeto, en este caso, los riesgos; por otro lado, se encuentran las normas y conceptualizaciones verificadas en las encuestas (método cuantitativo).

Este análisis permitió la clasificación de los riesgos y la determinación de los factores de riesgo encontrados en los laboratorios de práctica, para así determinar el panorama de riesgos propio del programa de bacteriología.

\section{Panorama de riesgos}

Una vez clasificados los riesgos, se elaboró el panorama según la norma Icontec, referenciando el riesgo, la fuente o agente, la consecuencia y el número de trabajadores, y valorando el factor de ponderación y la repercusión del riesgo, el tiempo de exposición y el grado de peligrosidad para evaluarlos teniendo en cuenta tres variables: consecuencia, exposición y probabilidad, e interrelacionándolas entre sí. Se analizaron los datos con los siguientes valores: de 0 a 300 , bajo, hay que mantener alerta y ejercer control; de 301 a 600 , medio, requiere corrección; de 601 a 1.000 , alto, exige una reacción inmediata; y mayor de 1.000 , muy alto, hay que paralizar las actividades (13-14).

Para la prevención frente al riesgo, se confrontó la normatividad establecida con los factores de riesgo detectados y se propusieron recomendaciones y observaciones como medidas de prevención a tener en cuenta frente a cada uno de ellos.

\section{Resultados}

\section{Levantamiento del diagnóstico}

De los 15 laboratorios estudiados, dos, el de micología y el de investigaciones, mostraron espacio pequeño pero buena organización, 
distribución y limpieza de los elementos y equipos de trabajo y con canecas adecuadas para los desechos; por tanto, no se realizó valoración de la contaminación.

El bioterio presentó grandes deficiencias en limpieza, distribución de elementos y organización y se concluyó que necesitaba un tratamiento especial.

El laboratorio central y sus 11 laboratorios satélites, donde se realizan las prácticas, mostraron algunas deficiencias en cuanto a espacios inadecuados, organización poco adecuada, distribución desordenada de elementos, limpieza deficiente de mesones, equipos y elementos insuficientes para desechos.

\section{Valoración de la contaminación}

Los cultivos practicados en la primera fase para detectar bacterias y hongos mostraron los siguientes resultados: en el laboratorio central, de los 24 cultivos practicados en ambiente, cepario, cuarto frío y cuarto de esterilización resultaron 16 $(68 \%)$ positivos para bacterias y $16(70 \%)$ para hongos. Las bacterias aisladas fueron Bacillus sp., Staphylococcus sp. y Micrococcus, y los hongos Penicillium sp., Aspergillus sp. y Mucor sp. En los laboratorios de práctica, de los 104 cultivos practicados en ambiente, vertederos, paredes, mesones y microscopios resultaron 57 (55\%) positivos para bacterias y $98(95 \%)$ para hongos.

En la segunda fase, los cultivos practicados para la detección de bacterias y hongos mostraron: en el laboratorio central, de los 24 cultivos practicados resultaron positivos $21(90 \%)$ para bacterias y 24 $(100 \%)$ para hongos; en los laboratorios de práctica, de los 104 cultivos se encontraron 104 $(100 \%)$ positivos para bacterias y $104(100 \%)$ positivos para hongos.

Las bacterias aisladas fueron Staphylococcus aureus, Staphylococcus sp. coagulasa negativa, Streptococcus B hemolítico y no hemolítico, Pseudomona sp. Klebsiella sp., E. coli y bacilos Gram positivos esporulados (cuadro 1, figura 2).

Los hongos aislados en las diferentes muestras fueron Penicillium, 90\%; Aspergillus, 90\%; Mucor, 40\%; levaduras, 20\%; Cladosporium, $30 \%$, y Fusarium, $30 \%$, en el laboratorio central. En los laboratorios de práctica, se encontró: Penicillium, 20\%; Cladosporium, $60 \%$; Rhizopus, 100\%; Mucor, $100 \%$; Scitadillium, 100\%; Fusarium, $100 \%$, y levaduras, $80 \%$ (cuadro 2, figura 3).

\section{Identificación y clasificación de los riesgos}

Riesgo biológico: de los resultados obtenidos con los tres métodos (observación participante, encuestas CAP y entrevista semiestructurada) se

Cuadro 1. Comparación de bacterias aisladas en los cultivos de la primera y la segunda fase.

Laboratorio central:

\begin{tabular}{lcc}
\hline Bacterias & $\begin{array}{c}\text { Fase 1 } \\
\%\end{array}$ & $\begin{array}{c}\text { Fase 2 } \\
\%\end{array}$ \\
\hline Cocos Gram (+) & 30 & 100 \\
Bacilos Gram (+) & 25 & 60 \\
Bacilos Gram (-) & 0 & 60 \\
\hline
\end{tabular}

Laboratorios de prácticas:

\begin{tabular}{lcc}
\hline Bacterias & $\begin{array}{c}\text { Fase 1 } \\
\%\end{array}$ & $\begin{array}{c}\text { Fase 2 } \\
\%\end{array}$ \\
\hline Cocos Gram (+) & 65 & 100 \\
Bacilos Gram (+) & 60 & 60 \\
Bacilos Gram (-) & 0 & 100 \\
\hline
\end{tabular}
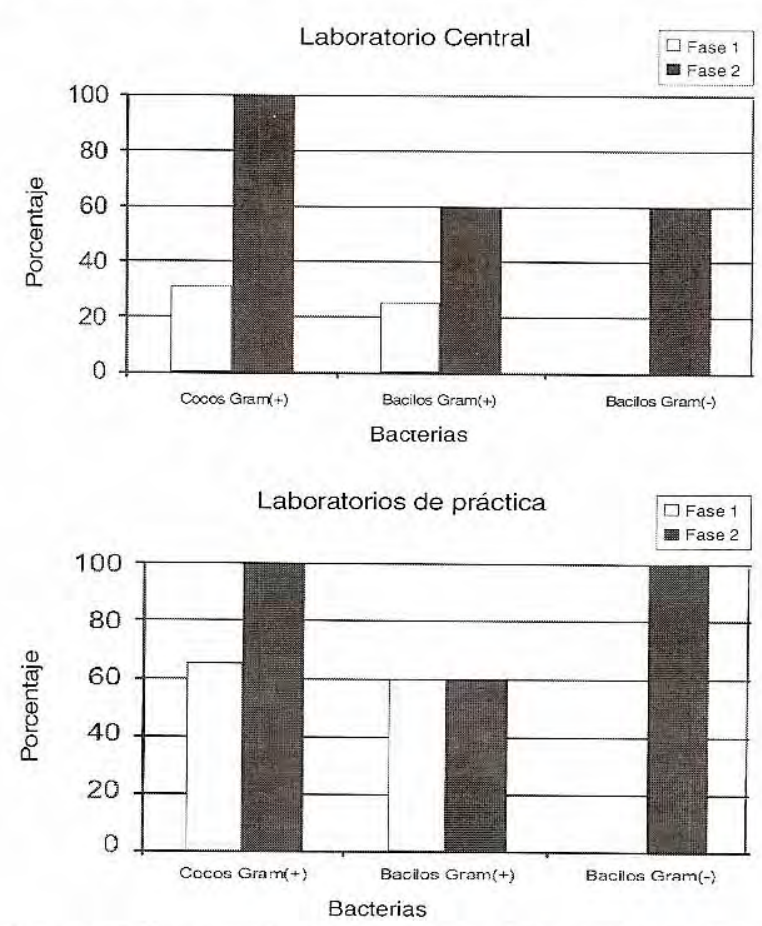

Figura 2. Comparación de bacterias aisladas en los cultivos de primera y segunda fase. 
Cuadro 2. Comparación de hongos aislados en cultivos en la primera y segunda fase.

Laboratorio central:

\begin{tabular}{lcc}
\hline Hongos & $\begin{array}{c}\text { Fase 1 } \\
\%\end{array}$ & $\begin{array}{c}\text { Fase 2 } \\
\%\end{array}$ \\
\hline Penicillium & 45 & 90 \\
Aspergillus & 30 & 90 \\
Mucor & 35 & 40 \\
Levaduras & 0 & 20 \\
Cladosporium & 0 & 30 \\
Fusarium & 0 & 30 \\
\hline
\end{tabular}

\begin{tabular}{lcc} 
Laboratorios de prácticas: & \\
\hline Hongos & Fase 1 & Fase 2 \\
& $\%$ & $\%$ \\
\hline Penicillium & 50 & 20 \\
Cladosporium & 60 & 60 \\
Rhizopus & 0 & 100 \\
Mucor & 0 & 100 \\
Scitadillium & 0 & 100 \\
Fusarium & 0 & 100 \\
Levaduras & 0 & 80 \\
\hline
\end{tabular}
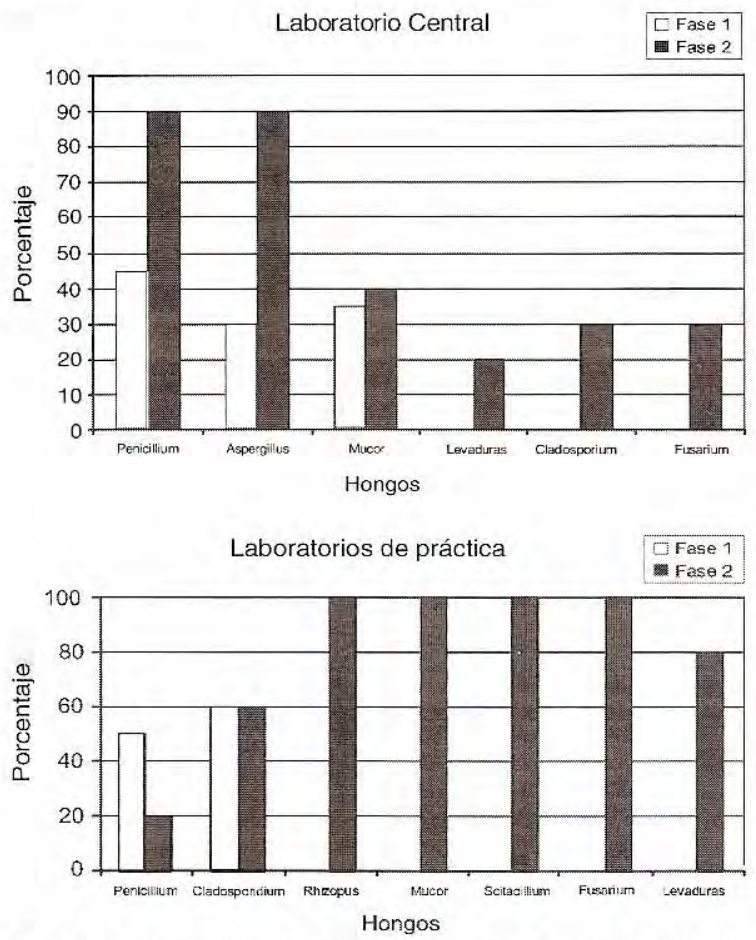

Figura 3. Comparación de los diferentes hongos aislados en los cultivos en la primera y segunda fase.

observó que los riesgos biológicos constituyeron el $80 \%$ como factor de riesgo ocupacional en la comunidad universitaria en los laboratorios de prácticas. Esta situación se relaciona con la manipulación de las muestras y de los microorganismos, en la que se notó una gran debilidad; así es como se encontró que $45 \%$ de la población tuvo dificultades en el manejo de las muestras para estudio microbiológico, $35 \%$ indicó deficiencia en el manejo de muestras sanguíneas y $20 \%$ en el manejo de otros líquidos corporales. El $80 \%$ de la población manifestó alto riesgo en el manejo de los desechos biológicos; igualmente en el $70 \%$ de la población hay desinformación sobre el manejo de animales de experimentación. En cuanto al uso de los elementos de barrera, se encontró que $30 \%$ los utilizaba en forma parcial. La desinfección de las áreas se realizaba adecuadamente en un $65 \%$ de los casos.

Riesgo psicosocial: el riesgo psicosocial se encontró en segundo lugar con un $70 \%$ de la población estudiada y estuvo relacionado con los comportamientos individuales frente al colectivo. Se observó que $70 \%$ de la población tenía presión en cuanto al manejo del tiempo. La responsabilidad de trabajar con agentes peligrosos, biológicos y químicos afectaba a $60 \%$ de la población. La mitad de los evaluados manifestó tener conflictos personales de diferente índole. Los conflictos del ambiente social incidian en $90 \%$ de la población. Entre estos conflictos sobresalieron problemas en el hogar, como desavenencias entre los padres, rivalidades, competencias y maltrato intrafamiliar. Dentro de los conflictos económicos, se encontraron el desempleo de los padres, la crisis económica y la pérdida del poder adquisitivo.

Riesgo químico: se encontró que $20 \%$ de los estudiados manejaban reactivos tóxicos y corrosivos; $40 \%$ conocía las normas específicas para el manejo de sustancias químicas y $80 \%$ de la población tenía dificultad con la información relacionada con los productos químicos.

Riesgo ergonómico: se observó que 25\% tenía dificultad en la manipulación y transporte de los microscopios; $15 \%$ se ve afectado por las posturas propias del desempeño.

Riesgo físico y de seguridad: el $90 \%$ de la población consideró fundamental el mantenimiento del sistema de gas y eléctrico y $20 \%$ se sintió 
afectado por la falta de ventilación o por la poca iluminación.

\section{Panorama de riesgos}

Riesgo biológico: de acuerdo con la fuente y el factor de riesgo clasificado, se obtuvo como resultado que las infecciones en general, las dermatitis, la contaminación, los accidentes, las heridas y laceraciones, valoradas mediante el grado de peligrosidad, estaban entre 400 y 144 con una repercusión del riesgo alto, mediano y bajo.

Riesgo psicosocial: las consecuencias de los factores de riesgo del grupo psicosocial se resumieron en comportamientos individuales, rendimiento, desinterés, fatiga, pasividad, poca iniciativa, depresión y baja concentración, que al ser valorados mediante el grado de peligrosidad estaban entre 600 y 216 con una repercusión del riesgo alto, mediano y bajo.

Riesgo químico: en este grupo, las consecuencias se relacionaron con quemaduras, intoxicaciones, irritación y escapes, que al ser valoradas mediante el grado de peligrosidad estaban entre 360 y 216 con una repercusión del riesgo entre medio y bajo.

Riesgo ergonómico: en este caso, las consecuencias se relacionaron con dolores de espalda, dolor lumbar, disminución visual, caídas, tropiezos, tensión muscular y cansancio, que al ser valoradas mediante el grado de peligrosidad alcanzaron en la escala hasta 216 con una repercusión de riesgo bajo.

Riesgo físico y de seguridad: para este grupo, se observaron como consecuencias quemaduras, riesgo de incendio, heridas por vidrio, caídas, errores en el trabajo y trastornos respiratorios, que al ser valorados mediante el grado de peligrosidad están entre 36 y 360 con una repercusión de riesgo entre medio y bajo.

Según la metodología seleccionada para el diseño del panorama de riesgo, se establecen recomendaciones o normas preventivas para cada uno de los factores de riesgo detectados.

En el riesgo biológico, se planteó la prevención frente al manejo de muestras, desinfección de las áreas físicas, laboratorios no especializados, utilización de equipos de protección personal, manejo y eliminación de desechos o residuos y de elementos cortopunzantes.

Para el riesgo psicosocial, la prevención se planteó para el exceso de trabajo, la tensión emocional, el estrés y las relaciones interpersonales.

En el caso del riesgo físico y de seguridad, la prevención se relacionó con el sistema de gas, el sistema eléctrico y la estructura física.

\section{Discusión}

Se conoce como riesgo ocupacional toda situación de trabajo que encierra una capacidad potencial de producir un accidente. Desde hace mucho tiempo, el hombre se ha preocupado por controlar los riesgos con los que tiene que convivir, ya sean los relacionados con energías que no regula o con las que aparentemente puede manejar. EI desarrollo tecnológico lleva relacionada muy intimamente la generación permanente de nuevos riesgos en todos los órdenes del quehacer cotidiano, con algunas paradojas que desconciertan a los dedicados al manejo y control de estos agresores. Con las ayudas tecnológicas, se suprimen algunos riesgos, pero salen a escena otros tal vez más contundentes y agresivos que los eliminados, como es el caso de la cantidad de residuos generados con el uso de materiales desechables (15).

No escapan a esta problemática las personas que trabajan en los laboratorios de salud, debido a la continua exposición a los diferentes riesgos que pueden afectar su salud y su seguridad (16). Hasta hace poco, la seguridad del trabajo se refería casi exclusivamente a los trabajadores de otras ocupaciones como minas, fundiciones, etc., y se prestaba poca atención a los accidentes del trabajo en los laboratorios de salud (1). Aparentemente, en estos laboratorios no suceden serios accidentes de trabajo ni enfermedades ocupacionales; sin embargo, al igual que en otros campos, los progresos de la ciencia han puesto de manifiesto lo vulnerables que pueden ser los trabajadores de los laboratorios de salud a los numerosos productos químicos peligrosos y a los variados y patológicos materiales biológicos que 
manejan, lo que ha permitido revelar la existencia de gran número de problemas de riesgo en esta actividad; por consiguiente, se considera que la protección de la salud y la seguridad del personal de los laboratorios es una acción obligante (1).

Las facultades de ciencias de la salud, como el caso que nos interesa en el presente estudio, son espacios comprometidos con toda esta problemática, por cuanto en su currículo se desarrollan prácticas de laboratorios de diferentes sectores, a saber: área de clínica con sus asignaturas de microbiología humana y veterinaria, bioquímica, hematología, inmunología y parasitología, y otras con características muy semejantes a las de los laboratorios clínicos de los hospitales que, desde el punto de vista de las normas de la Secretaría de Salud, están catalogados como áreas críticas de mucho riesgo (17).

Así mismo, otras áreas del programa de bacteriología también generan riesgo como son los laboratorios donde se realizan prácticas de industrial, química, toxicología y otros de este campo. Toda esta situación involucra una serie de responsabilidades que se inician con la clasificación de los riesgos propios de cada institución, que permita diseñar un panorama de riesgos específico y, a su vez, puntualizar las medidas de prevención que se deben aplicar para minimizar los riesgos en el quehacer diario.

En el presente estudio se observó la presencia de patógenos importantes como $S$. aureus, $S$. pyogenes, Pseudomona sp., levaduras y hongos en gran cantidad como Penicillium sp., Aspergillus sp., Mucor sp. y Scitadillium sp., que ponen de presente la deficiencia en las prácticas de bioseguridad, lo que facilita que estos patógenos se dispersen. Los estudios relacionados con contaminación ambiental en los laboratorios permiten apreciar la variedad de microorganismos que pueden encontrarse en un medio ambiente cuando las normas de bioseguridad no se cumplen (8). Se encontraron muchos de los patógenos que, generalmente, se encuentran como contaminantes ambientales.

Es importante tener en cuenta normas específicas para el manejo de microorganismos que eviten su dispersión; las fallas en su aplicación conllevan a la contaminación por formación de aerosoles, accidentes por derrames de material biológico, infecciones por manipulación de cultivos y laceraciones con elementos cortopunzantes (18).

En el panorama de riesgos producto de esta investigación, se aprecia cómo el biológico fue el primer factor de riesgo para los laboratorios de diagnóstico del programa de bacteriología, situación que se encuentra acorde con otros estudios como el realizado en la Universidad de San Pablo (19), el cual puntualiza que el logro de los objetivos de prevención, protección y seguridad en el trabajo es tarea de conjunto en la que están involucrados todos los usuarios de las diversas áreas de una institución, desde los órganos de dirección hasta los trabajadores que participan en el funcionamiento de la universidad, pasando por los alumnos de cualquiera de los cursos que en ésta se imparten. Del conocimiento de sus obligaciones y del estricto cumplimiento de las normas básicas establecidas, dependerá la disminución del riesgo y, por tanto, la posibilidad de evitar accidentes.

En el caso del programa de bacteriología, se apreció que hay buen conocimiento de la norma, pero dificultad en la aplicación permanente de la misma. Es así como dentro de la escolaridad y formación de las profesionales en bacteriología, se enseñan y enfatizan tanto las precauciones universales como las medidas de bioseguridad específicas a través del desarrollo de las prácticas, según las normas establecidas por la Organización Panamericana de la Salud y el Ministerio de Salud; sin embargo, como se observó en los resultados de la valoración de la contaminación ambiental hay vacíos en la aplicación de las normas que es indispensable corregir para lograr hábitos y comportamientos que conlleven a una cultura de la prevención.

De igual manera, en el trabajo realizado en el Instituto de Medicina Tropical Pedro Kouri de Cuba sobre riesgo biológico ocupacional, en la sección de medidas de seguridad en los laboratorios médicos se concluye que el aislamiento o contención como medio necesario para preservar la esterilidad de los cultivos de microorganismos 
y de células es un requisito reconocido por los científicos desde hace muchos años, pero sólo cuando comenzaron a aparecer casos de infecciones adquiridas se prestó atención a la protección personal de los trabajadores de los laboratorios (20).

En los últimos 25 años, el número de personas empleadas en los laboratorios se ha incrementado considerablemente y, por ende, la preocupación por los riesgos de infección del personal de laboratorios y por intensificar las exigencias con respecto a los niveles de bioseguridad. Periódicamente se realizan reuniones de comisión de expertos para actualizar la información y definir políticas y recomendaciones con respecto a la seguridad de los laboratorios e identificar las exigencias con respecto a los niveles de bioseguridad (21).

El segundo factor de riesgo detectado en este estudio fue el riesgo psicosocial resultante de factores como el exceso de trabajo, la tensión emocional, las relaciones interpersonales y el estrés. Según el estudio realizado en el Instituto Pedro Kouri, adicionalmente existe un grupo de riesgos fundamentales constituido por factores humanos, los cuales pueden incrementar considerablemente la incidencia de los otros factores y pueden estar relacionados con las aptitudes y habilidades para el trabajo, el estado físico y psicológico del trabajador, su capacidad intelectual y entrenamiento laboral, así como la organización general del laboratorio (20).

El nivel de sensibilidad y acción que se logre en todos aquellos que, de una u otra forma, tienen que ver con el trabajo en los laboratorios de diagnóstico, determinará el éxito en la implementación de las medidas de seguridad y su eficiencia en la prevención y limitación de los efectos perjudiciales.

En tercer lugar se identificó el riesgo químico, contrario a lo que se esperaba, ya que siempre se piensa que el mayor riesgo en laboratorios es por las sustancias tóxicas que se manejan (22). En nuestro caso, hay razones fundamentales que minimizan la exposición a este riesgo. En primera instancia, el tiempo de exposición es corto, en ocasiones sólo dos a tres horas a la semana. Así mismo, los reactivos utilizados son de mediana peligrosidad y se usan en pequeñas cantidades; de otro lado, la tecnología ha evolucionado y las pruebas bioquímicas que podían ser generadoras de mucho riesgo se han cambiado por pruebas preparadas (en su mayoría, estuches con reacciones enzimáticas) que son, generalmente, inocuos o de bajo riesgo. Sin embargo, se apreció la necesidad de reforzar el conocimiento acerca del manejo y desecho de ácidos, bases y alcoholes.

En el estudio realizado en la Facultad de Ciencias Químicas de la Universidad Complutense de Madrid, se recomienda que para trabajar con sustancias químicas hay que tener un conocimiento preciso (23). Antes de realizar cualquier operación de la que no se esté completamente seguro, se preguntará al profesor de práctica o responsable del laboratorio. Toda persona que maneje un producto químico deberá conocer sus características fisicoquímicas y su toxicidad. Así mismo, la eliminación de los productos químicos se debe realizar de acuerdo con la norma para evitar contaminación del medio ambiente.

El personal que labora en los laboratorios de diagnóstico está sujeto a gran cantidad de riesgos de peligrosidad variable y de causas multivariadas en las que intervienen varios factores como la responsabilidad individual del trabajador y responsabilidades colectivas y administrativas porque desempeñan funciones preponderantes o coadyuvantes en la ocurrencia de accidentes (24).

Como solución a la problemática que se observó en el diagnóstico de los factores de riesgo identificados y para apoyar las políticas del programa de salud ocupacional institucional se plantea el programa educativo para la prevención de los riesgos en las prácticas de laboratorios del programa de bacteriología (11).

Esta investigación permitirá que la comunidad universitaria reflexione y se cuestione acerca de la presencia de los riesgos en el quehacer cotidiano y así mismo involucre en su formación la cultura de prevención frente al riesgo de todas y cada una de las actividades. 


\section{Agradecimientos}

A la Universidad Colegio Mayor de Cundinamarca y a sus directivas por el apoyo para el desarrollo de esta investigación; a la comunidad educativa por su colaboración y aportes, y a las personas que con gran dedicación y espontaneidad aportaron sugerencias a este manuscrito.

\section{Referencias}

1. Moreno RA. Problemática de los riesgos laborales. Protección y seguridad 1990;(3):17-21.

2. Cerquera MT. Proponer la salud. Asesoria de educación para la salud y participación social. Washington D.C. OPS/OMS; 1992. p.19-25.

3. OPS. Manual de encuestas sobre conocimientos, actitudes, creencias y prácticas ETS y SIDA. Washington D.C.: Oficina Sanitaria Panamericana; 1993.

4. Wiley J. Introduction to quantitative research methods The search for meanings. Madrid, España: Nova-Grafik, S.A.; 1992. p.158-220.

5. Rostagno H. Conocimiento, estudio y prevención actualizado. Revista Empresa Salud (seriado en línea) 1997. Disponible en http://www.hq.satlink.com/ empresasalud/revista32n-am-2hHm.

6. Best M, Kennedy M. Sterilization and desinfection of instruments and materials in the surgery unit and laboratory areas. Semin Dermatol 1995;14:243-6.

7. OMS. Laboratory biosafety manual. Second edition. Geneve: World Health Organization 1993; p-15-22.

8. Gersbon R. Bilological safety Isenberal. In: Isenberg $H D$, editor. Clinical microbiology procedures handbook. Washington, D.C.: American Society of Microbiology; 1992. p.141.1-141.6.

9. Andrade S, Shedlin M. Métodos cualitativos para la evaluación de programas. Bogotá: Editorial Magisterio; 1990. p.45-97.

10. Bonilla E, Andrade S, Shedlin M. Métodos cualitativos para la evaluación de programas. Bogotá: Editorial Magisterio; 1992. p.22-135.

11. Ministerio de Salud. Decreto No. 1891. Fomento de la salud y prevención de la enfermedad, 1994, Ley 60 de 1993, Ley 1298 de 1994.

12. Ministerio de Educación Nacional. La educación en salud en el proyecto educativo nacional. Serie de documentos de trabajo. Santa Fe de Bogotá: Ministerio de Educación Nacional; 1996.

13. Donalisio R. Confección de mapas de riesgos en la compañia metropolitana en riesgos de Madrid. Propuesta metodológica. Protección y Seguridad 1990;(2):15-25.

14. Moreno RA. Panorama general de riesgos ocupacionales en el sector metalúrgico. Seminario taller. Bogotá: Fedemetal, CCS; 1990. p.75-82.

15. Sewell D. Laboratory associated infection and biosafety. Clin Microb Rev 1995;8:389-95.

16. Truchand $\mathbf{S}$. Increasing the biosafety of analytical system in the clinical laboratory. Ann Biol Clin Paris 1994;52:393-6.

17. Ministerio de Salud. Conductas básicas en seguridad. Manejo integral. Protocolo básico para el equipo básico de salud. Santa Fe de Bogotá: Ministerio de Salud; 1997. p.19-25.

18. Stevenson R. Applications of good laboratory practice to culture collections of microbiology and cell cultures. $J$ Microbiol Biotechnol 1992;8:229-35.

19. Universidad de San Pablo, CEU, Facultad de Ciencias Experimentales y Técnicas, Unidad de prevención y riesgos laborales. Normas básicas de seguridad social en los laboratorios. Revistas electrónicas EBSCO on line 1990. Disponible en http:// www.ceu.es/ceu/cnt/mad/usp/biblio.htm

20. Fernández L, De la Cruz C. Riesgo biológico, ocupacional y medidas de seguridad en los laboratorios médicos. Instituto de Medicina Tropical Pedro Kouri. CEPIS, 1999. Disponible en http://www.cepis.ops-oms.org/ eswww/fulltext/repind61/rboms/m/rboms.htm

21. Ministerio de Trabajo y Seguridad Social. Estudio y análisis del riesgo biológico ocupacional en Colombia. Informe técnico. Bogotá: Ministerio de Trabajo y Seguridad Social; 1994. p.7-10.

22. Merck. Manual para el laboratorio - Alemania. Segunda edición. Madrid, España: Merck Sharp Dohme de Esapaña; 1995. p.70-85.

23. Universidad Complutense de Madrid. Facultad de Ciencias Químicas. Documento sobre recomendaciones generales para la seguridad de personas e instalaciones. España. Revistas electrònicas de química on line, 1990. Disponible en http://alfama.sim.uvm.es/BUC/ nueaccesotelefonico.htm

24. Ferrada L. Riesgos ocupacionales en hospitales. Protección y Seguridad 1990;(3):44-7. 